\title{
QUASICLASSICAL MODEL OF MANY-ELECTRON QUANTUM DOTS *
}

\author{
E. Anisimovas and A. Matulis \\ Semiconductor Physics Institute, A. Goštauto 11, LT-01108 Vilnius, Lithuania \\ E-mail: egidijus@pfi.lt, amatulis@takas.lt
}

Received 25 July 2005

\begin{abstract}
The general quasiclassical description of parabolic many-electron quantum dots in the limit of high magnetic fields is presented. We obtain the complete wave function of quantum dots containing an arbitrary number of electrons in the form of a Gaussian function and calculate the electron-electron correlation function. Using this function we describe the Wigner crystallization in quantum dots emphasizing effects brought about by the finite size of the dots.
\end{abstract}

Keywords: quantum dots, Wigner crystallization, quasiclassical approximation

PACS: 73.21.La, 71.10.-w

\section{Introduction}

Probably the best definition of a quantum dot is 'artificial atom' [1-3]. This popular nickname is justified by the fact that quantum dots confine a small number of electrons to a limited space and feature sharply quantized energy spectra as well as the shell structure [4] characteristic of natural atoms. An obvious advantage of artificial atoms over their natural counterparts is the much grater controllability of their structure and composition.

In particular, visualizing a quantum dot as a confining 'box' for electrons of characteristic dimension $l$ one quickly realizes that the characteristic energy of the inter-electron Coulomb interactions scales as $\sim l^{-1}$ whereas the quantum-mechanical kinetic energy is proportional to $l^{-2}$. Thus, by just making a quantum dot bigger one may switch it into a regime where the relative role of Coulomb correlations is overwhelming. Under these circumstances crystallization of electrons into a solid-like structure - known as the Wigner [5] crystallization - is expected to occur. Such crystals were identified from electrical conductance measurements [6] in two-dimensional electron layers.

As a matter of fact, variation of the size of a quantum dot is cumbersome and in fact unnecessary. The enhancement of the relative importance of inter-electron Coulomb correlations may be more easily brought

\footnotetext{
${ }^{*}$ The report presented at the 36th Lithuanian National Physics Conference, 16-18 June 2005, Vilnius, Lithuania.
}

about by an application of a perpendicular magnetic field [7]. The field forms a ladder of Landau levels separated by large (proportional to the magnetic field $B)$ gaps. At zero or very low temperatures and strong magnetic fields all electrons accumulate on the lowest Landau level and the inter-Landau-level motion is effectively frozen out. The dynamics is dominated by the Coulomb interactions, and such a system is essentially classical. The quantum mechanical kinetic energy may be completely disregarded in the limit of extremely strong magnetic fields, and taken into account perturbatively at somewhat weaker fields. These circumstances have encouraged us to treat quantum dots in a quasiclassical way basing on the expansion in inverse powers of the applied magnetic field [8-10]. The complete quantum-mechanical solution, on the other hand, is computationally demanding $[11,12]$ and thus must be restricted to systems of a few electrons.

In the present paper we apply the quasiclassical theory [10] to describe the formation of Wigner crystallites and shell structure in quantum dots. We obtain the many-electron wave function and the density-density correlation function. These functions reveal that the structure and properties of the Wigner molecule are set by the competition of symmetries - hexagonal crystal lattice and isotropic confinement.

The quasiclassical theory and computational procedure are briefly reviewed in Sect. 2, and Sect. 3 presents the results. 


\section{Model}

Our model of quantum dots features $N$ two-dimensional electrons laterally confined by a circularly symmetric parabolic trap.

The computations are carried out in two steps. First, we look at the limit of extreme magnetic fields where the kinetic energy may be neglected. Thus, the problem reduces to the minimization of the total (confinement plus Coulomb interaction) energy of $N$ twodimensional electrons in a parabolic confinement

$$
V=\frac{1}{2} \sum_{i=1}^{N} r_{i}^{2}+\sum_{i=1}^{N} \sum_{j>i}^{N} \frac{1}{\left|\boldsymbol{r}_{i}-\boldsymbol{r}_{j}\right|} \rightarrow \min
$$

written in terms of scaled dimensionless coordinates.

This problem is solved numerically using Monte Carlo simulations [13]. Starting from a random initial configuration, each electron in turn is allowed to make a small step in a random direction. This step is accepted, and the configuration updated, if it leads to a lowering of the total potential energy $V$, or rejected otherwise. As a matter of fact, this procedure leads to finding the nearest local minimum and not necessarily the global one. The number of local minima rapidly grows with the number of electrons, thus, in order to avoid the system getting trapped in a local minimum it is necessary to modify the algorithm and with a certain probability also to accept steps that increase the total energy. This procedure, loosely speaking, corresponds to simulated dynamics at an elevated temperature which allows the system to overcome energy barriers surrounding local minima of the multidimensional potential landscape.

Figure 1 illustrates the results of such a simulation performed for several electron numbers up to 19. Already in these results, the general trends of the formation of the shell structure are visible. Up to five electrons can be accommodated on a single ring. Increasing the number of electrons, some of them penetrate the interior of the outer ring and form an inner one while the remaining ones find place in the expanded outer shell. This scenario is repeated over and over again, and for example in 19-electron quantum dot we find three shells containing 1,6 , and 12 particles, respectively.

Another important piece of information extractable from the results of classical Monte Carlo simulations is the second order expansion of the potential energy around the equilibrium configuration

$$
V=V^{(0)}+V^{(2)}(\{x, y\}) .
$$

Here, $V^{(0)}$ denotes the total potential energy at the equilibrium, the first-order term is absent by definition, and $V^{(2)}$ is a quadratic form in the coordinates of all electrons symbolically presented as the set $\{x, y\}$. In an ordinary vibrational problem of classical mechanics, the eigenvalues of the quadratic form matrix give the squared oscillation frequencies of all eigenmodes. Our problem to construct the quasiclassical theory is somewhat more complicated and a more careful analysis is needed.

At finite magnetic fields, the contribution due to the kinetic energy must be taken into account, and the predominant type of dynamics is indeed small oscillations of electrons around the equilibrium positions. Under the influence of the strong magnetic field the motion of electrons resembles circulation around the crystallization point rather than ordinary vibrations [9].

In a circularly symmetric potential, which is used in our model, the quantum dot will also support a rotational mode which may couple with the vibrational modes [14] and has to be carefully separated. It is interesting to observe that this issue was already encountered in molecular physics when treating the rotovibrational motion of molecules [15].

In the rotational mode the Wigner molecule rotates as a whole. That is, all the electrons move with velocities proportional to their distance from the centre of the quantum dot in the azimuthal direction. As a matter of fact, parabolic quantum dots support another symmetric special mode - the so-called breathing mode. In this mode all the electrons also move with velocities proportional to their distances from the dot centre in the radial direction. The breathing mode is a universal separable mode in parabolic confinement [10].

Having separated the special modes we are left with the vibrational problem. We succeeded in writing the vibrational Hamiltonian in a compact form. Before presenting it we first introduce a column-vector collecting all the generalized collective coordinates

$$
w=\left(\begin{array}{c}
w_{1} \\
w_{2} \\
\cdots \\
w_{2 N-2}
\end{array}\right)
$$

There are $2 N-2$ of them since two special modes have been eliminated. The coordinates $w_{i}$ are chosen orthogonal to the breathing and rotational modes. For the sake of convenience the coordinates $w_{i}$ are ordered in a definite way. The first $N-1$ of them are linear 

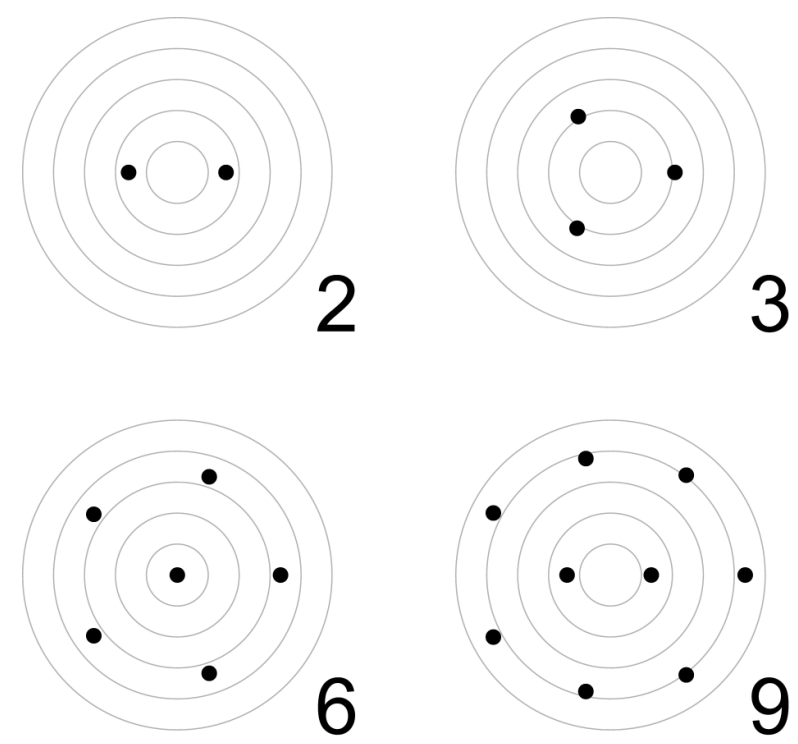
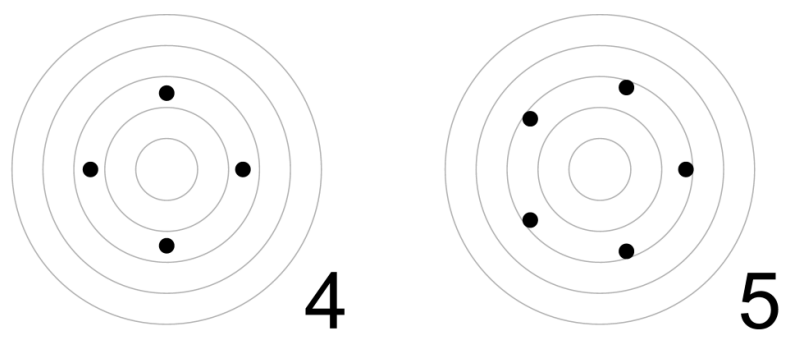

5

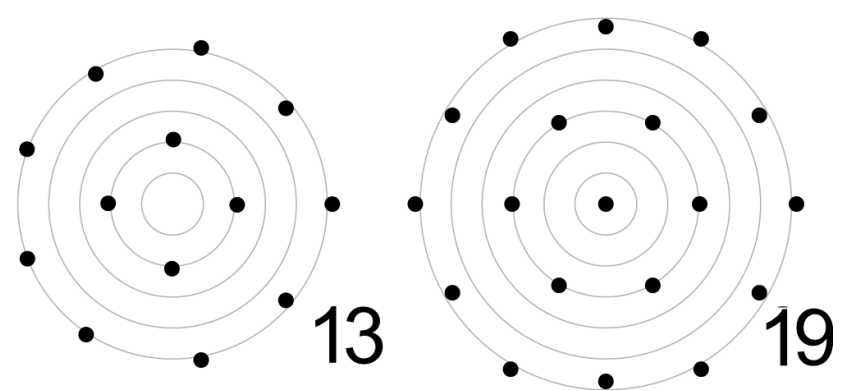

Fig. 1. Monte Carlo simulation. The black dots mark the equilibrium positions of the electrons and the concentric rings are drawn to portray the shape of the confining potential.

combinations of the radial displacements of electrons from the equilibrium, and the second batch is formed by identical linear combinations of the azimuthal displacements. The corresponding vector of momenta is

$$
W=\left(\begin{array}{c}
\frac{\partial}{\partial w_{1}} \\
\frac{\partial}{\partial w_{2}} \\
\cdots \\
\frac{\partial}{\partial w_{2 N-2}}
\end{array}\right)
$$

In order to write the vector products in a matrix notation we also define an auxiliary $(2 N-2) \times(2 N-2)$ matrix

$$
\mathcal{G}=\left(\begin{array}{cc}
0 & -\mathrm{i} \mathcal{I} \\
\mathrm{i} \mathcal{I} & 0
\end{array}\right)
$$

with $\mathcal{I}$ being $(N-1) \times(N-1)$ unity matrix, and obtain the vibrational Hamiltonian in the form

$$
H_{\mathrm{vib}}=\frac{1}{2}\left(W-\frac{\mathrm{i} B}{2} \mathcal{G} w\right)^{2}+\frac{1}{2} w^{T} V^{(2)} w .
$$

The first part of the Hamiltonian (6) has the form of the kinetic energy of a system of $N-1$ charged particles in a magnetic field, and the second part is the quadratic form of the potential expressed in terms of the collective coordinates $w$.
The ground state of the parabolic Hamiltonian (6) is given by a Gaussian function, that is, an exponentiated quadratic form

$$
\Psi_{\mathrm{vib}}=\exp \left(-\frac{B}{4} w^{T} \mathcal{X} w\right)
$$

with a so far unknown matrix $\mathcal{X}$. Inserting (7) into the Hamiltonian (6) we express the energy

$$
E=\frac{B}{4} \operatorname{Tr} \mathcal{X}
$$

in terms of the trace of the matrix $\mathcal{X}$, and obtain an equation for $\mathcal{X}$

$$
\mathcal{X}^{2}+\mathcal{G} \mathcal{X}-\mathcal{X} \mathcal{G}-\mathcal{I}-\frac{4}{B^{2}} V^{(2)}=0 .
$$

Note that this is a quadratic matrix equation, also known as the Riccati equation. We solved [10] this equation using a standard method based on the introduction of an auxiliary supermatrix containing the coefficients of (9) as its blocks and on the solution of its spectral problem.

\section{Results}

The knowledge of the many-electron wave function allows us to calculate the distribution of the charge density in the quantum dot. However, this distribution retains the circular symmetry of the Hamiltonian and does not enable one to study the internal structure of the Wigner crystallite. In other words, in a circularly symmetric confinement the orientation of the 

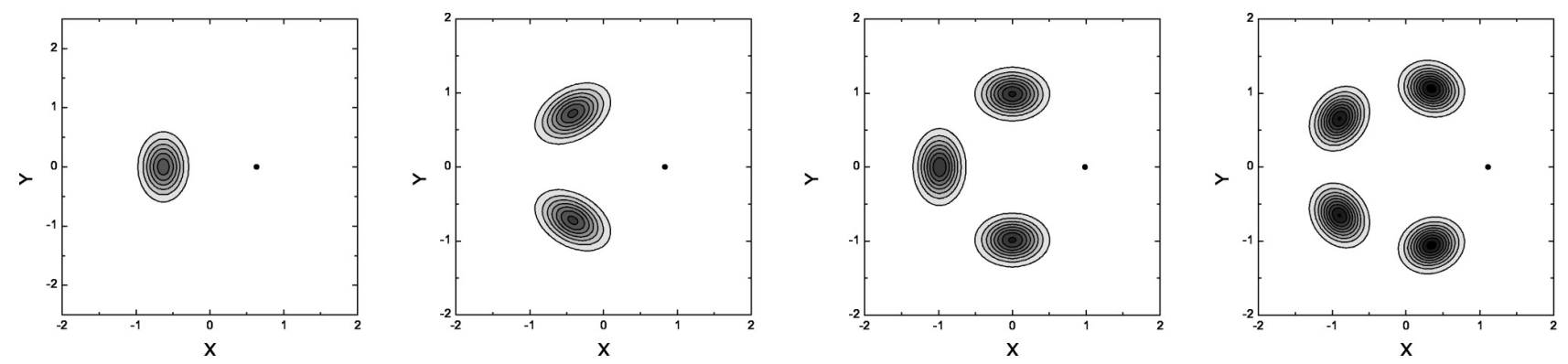

Fig. 2. Contour plots of the conditional probability densities of single-shell quantum dots, containing 2 to 5 electrons. In each frame, black dots mark the position of the pinned electron.

formed Wigner crystal is not fixed. The crystal can rotate around its axis thus hiding its structure.

In order to study the crystallization one must invoke the correlation functions that may be interpreted as conditional densities given that one electron of a manyparticle system is pinned down at a given point. We always choose to pin one of the electrons in its equilibrium position and place it on the positive part of the $x$ axis. In the figures below, these positions are marked by dots.

Figure 2 shows the correlation functions of quantum dots containing between 2 and 5 electrons. One of the electrons is pinned on the positive part of the $x$ axis at the distance equal to the classical ring radius from the centre. The remaining electrons crystallize around their equilibrium positions forming a regular polygon. We see that the conditional charge density lumps are more extended in the azimuthal direction and compressed in the radial direction. That is, the electrons are more free to vibrate along the circumference of the ring than from/to the centre. However, as the number of electrons increases, the charge density lumps become more and more rounded indicating the restricted easiness of the azimuthal motion as well. Five electrons is the max- imum number that can be accommodated on a single ring.

Figure 3 shows that the sixth electron falls into the centre of the ring, thus increasing the radial repulsion of the remaining electrons and enlarging the ring radius. Consequently, the seventh and eighth electrons can again find their equilibrium positions on the ring. As before, we see that the charge density lumps are extended in the azimuthal direction but less so for a greater number of electrons.

For electron numbers equal to nine and larger, a true inner ring - rather than just a single electron in the centre - is formed. The correlation functions indicate a rather weak correlation between the rings. For 9, 10, and 11 electrons the inner ring is seen to rotate rather freely with respect to the outer one. When an electron in the outer shell is pinned the constant density ellipses on the inner ring are strongly elongated in the azimuthal direction. It is interesting to observe that this fact correlates with the presence of a soft (low frequency) classical mode.

This tendency, however, is interrupted in the twelveelectron quantum dot. Here we see that the ellipses on the inner electron ring are only slightly elongated in the
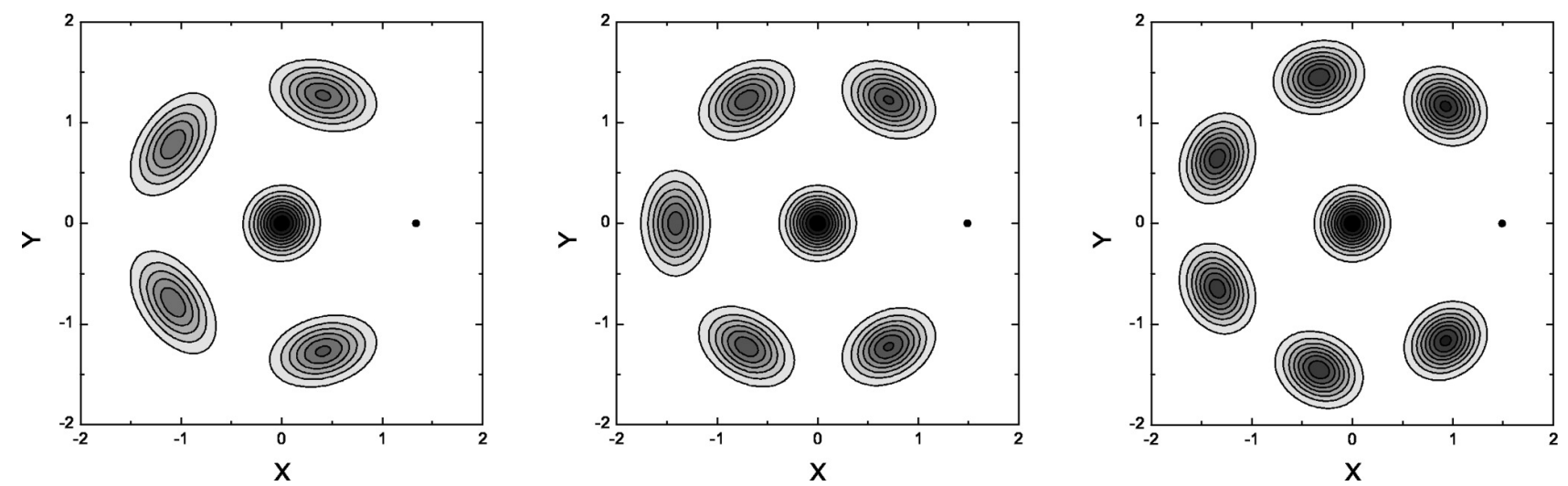

Fig. 3. Contour plots of the conditional probability densities of quantum dots containing 6 to 8 electrons. In each frame, the position of the pinned electron is marked by a black dot. One of the free electrons is placed into the centre while the remaining ones arrange on the corners of a regular polygon. 

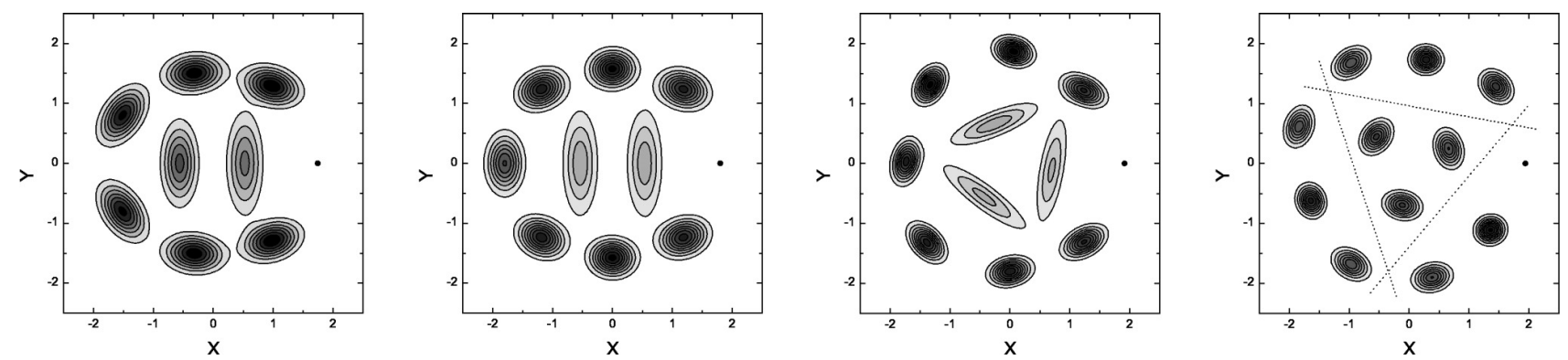

Fig. 4. Contour plots of the conditional probability densities of quantum dots containing between 9 and 12 electrons. In each frame, the black dot marks the position of the fixed electron. An inner shell containing 2 or 3 electrons is formed which can rotate with respect to the outer one relatively easily, except for the 12-electron dot having a nearly triangular structure (made more apparent by the dotted lines).

azimuthal direction, indicating a rather strong coupling between the rings. This can be explained by commensurability of electron numbers in the two shells - three plus nine. For this combination electrons organize into a triangular structure (emphasized by the dotted lines in Fig. 4) compatible with the hexagonal lattice preferred by Wigner crystals in extended two-dimensional systems.

\section{Acknowledgement}

This work was supported by the Lithuanian Science and Studies Foundation under grant No. T-05/18.

\section{References}

[1] P.A. Maksym, H. Imamura, G.P. Mallon, and H. Aoki, J. Phys.: Condens. Matter 12, R299 (2000).

[2] L.P. Kouwenhoven, D.G. Austing, and S. Tarucha, Rep. Prog. Phys. 64, 701 (2001).

[3] S.M. Reimann, Rev. Mod. Phys. 74, 1283 (2002).
[4] S. Tarucha, D.G. Austing, T. Honda, R.J. van der Hage, and L.P. Kouwenhoven, Phys. Rev. Lett. 77, 3613 (1996).

[5] E. Wigner, Phys. Rev. 46, 1002 (1934).

[6] H.W. Jiang, R.L. Willett, H.L. Stormer, D.C. Tsui, L.N. Pfeiffer, and K.W. West, Phys. Rev. Lett. 65, 633 (1990).

[7] A. Matulis and F.M. Peeters, Solid State Commun. 117, 655 (2001).

[8] E. Anisimovas, A. Matulis, M.B. Tavernier, and F.M. Peeters, Phys. Rev. B 69, 075305 (2004).

[9] E. Anisimovas, A. Matulis, and F.M. Peeters, Phys. Rev. B 70, 195334 (2004).

[10] A. Matulis and E. Anisimovas, J. Phys.: Condens. Matter 17, 3851 (2005).

[11] S.A. Mikhailov, Phys. Rev. B 65, 115312 (2002).

[12] M.B. Tavernier, E. Anisimovas, F.M. Peeters, B. Szafran, J. Adamowski, and S. Bednarek, Phys. Rev. B 68, 205305 (2003).

[13] V.M. Bedanov and F.M. Peeters, Phys. Rev. B 49, 2667 (1994).

[14] P.A. Maksym, Phys. Rev. B 53, 10871 (1996).

[15] C. Eckart, Phys. Rev. 47, 552 (1935).

\title{
KVAZIKLASIKINIS DAUGIAELEKTRONIŲ KVANTINIŲ TAŠKŲ MODELIS
}

\author{
E. Anisimovas, A. Matulis \\ Puslaidininkiu fizikos institutas, Vilnius, Lietuva
}

\section{Santrauka}

Pristatoma kvaziklasikinė teorija, aprašanti daugiaelektronius parabolinius kvantinius taškus stipriuose magnetiniuose laukuose. Pagrindinès būsenos daugiadalelinė banginè funkcija užrašoma
Gauso funkcijos pavidalu ir iš jos gaunama elektronų koreliacinè funkcija, aprašanti Wigner'io kristalizaciją. Atskleidžiama simetrijų konkurencija tarp apskrito kvantinio taško sieneliu potencialo ir šešiakampès vidinès Wigner'io kristalo sandaros. 